\title{
MHD simulations of coronal dark downflows considering thermal conduction
}

\author{
E. Zurbriggen ${ }^{1}$, A. Costa $^{1,2}$, A. Esquivel ${ }^{3}$, M. Schneiter ${ }^{1,2}$ \\ and M. Cécere ${ }^{1}$ \\ ${ }^{1}$ Instituto de Astronomía Teórica y Experimental, CONICET, Córdoba, Argentina \\ ${ }^{2}$ Facultad de Ciencias Exactas, Físicas y Naturales, UNC, Argentina \\ ${ }^{3}$ Instituto de Ciencias Nucleares, Universidad Nacional Autónoma de México, México
}

\begin{abstract}
While several scenarios have been proposed to explain supra-arcade downflows (SADs) observed descending through turbulent hot regions, none of them have systematically addressed the consideration of thermal conduction. The SADs are known to be voided cavities. Our model assumes that SADs are triggered by bursty localized reconnection events that produce non-linear waves generating the voided cavity. These subdense cavities are sustained in time because they are hotter than their surrounding medium. Due to the low density and large temperature values of the plasma we expect the thermal conduction to be an important process. Our main aim here is to study if it is possible to generate SADs in the framework of our model considering thermal conduction. We carry on 2D MHD simulations including anisotropic thermal conduction, and find that if the magnetic lines envelope the cavities, they can be isolated from the hot environment and be identified as SADs.
\end{abstract}

Keywords. Sun: corona, magnetic fields, Physical Processes: MHD, conduction, instabilities, turbulence, shock waves

\section{Introduction}

Supra-arcade downflows (SADs) are dark zigzag-moving patterns descending from $\sim 40-60 \mathrm{Mm}$ above solar arcades, with speeds of $\sim 50-500 \mathrm{~km} \mathrm{~s}^{-1}$ and sizes of $\sim 1-10$ Mm (McKenzie \& Savage 2009; Savage \& McKenzie 2011). Because of SADs' lack of emission (in soft X-ray, EUV and UV radiation) in images and spectra, it is well known that they are subdense cavities and not cool absorbing material (Innes et al. 2003). SADs' detections occur during eruptive events associated with coronal mass ejections (Asai et al. 2004) in the region above the arcade, which is a hot turbulent environment called fan (McKenzie 2013). A fan, with temperature $(\sim 10 \mathrm{MK})$ and density $\left(\sim 10^{-14} \mathrm{~g}\right.$ $\mathrm{cm}^{-3}$ ) approximately one order of magnitude higher than the usual quiet corona, and which is supposed to envelope a current sheet (CS) as a sort of thermal halo (Hanneman \& Reeves 2014), is heated and expanded due to the heat flowing from the hot CS.

As we expect the thermal conduction (TC) is an efficient process in fan, we believe is important to take it into account to explain SADs' origin and dynamics. While several models of SADs were proposed (Costa et al. 2009; Linton et al. 2009; Savage et al. (2012); Guo et al. 2014), the explicit consideration of TC has generally been neglected. Within our framework we assume that SADs are triggered by bursty localized reconnection events which occur during longer term eruptive processes (Costa et al. 2009) in inhomogeneous fans (Cécere et al. 2012; and in Cécere et al. 2015, hereafter Paper I). We modeled the bursty reconnection processes as blasts emulating localized depositions of energy released in short times, then leading to the development of shocks and rarefaction waves that produce subdense cavities (Paper I). 
Table 1. Parameters of the simulated stages

\begin{tabular}{lccccccc}
\hline Stages & $T[\mathrm{MK}]$ & $\rho\left[\mathrm{g} \mathrm{cm}^{-3}\right]$ & $\mathbf{B} \cdot \hat{\mathbf{j}}[\mathrm{G}]$ & $|\mathbf{v}|\left[c_{s}\right]$ & Stir & TC & Shear \\
\hline Turb & 6.0 & $1.2 \times 10^{-14}$ & 3.0 & 0.0 & yes & no & no \\
\hline Fan & $\langle 7.0\rangle$ & $\left\langle 1.2 \times 10^{-14}\right\rangle$ & $\langle 3.0\rangle$ & $\langle 0.21\rangle$ & no & yes & yes \\
\hline
\end{tabular}

Note. Parameter values for the different simulated stages. Second row: homogeneous initial conditions of the simulation and of the turbulent stage. Third row: initial conditions of the fan stage, i.e. the plasma parameters once the turbulent stage is completed (framework parameters for the 2D simulations). The initial density $\rho$ is equivalent to $n_{i}=5 \times 10^{9} \mathrm{~cm}^{-3}$. The quantities in $\langle\ldots\rangle$ means average over all grid cells. At the initial fan stage $\left\langle c_{s}\right\rangle=4.0 \times 10^{7} \mathrm{~cm} \mathrm{~s}^{-1}$.

The TC is anisotropic where the heat flux is mostly funneled along magnetic field lines and strongly inhibited across them (Spitzer 1962). Moreover, even in plasmas subject to high temperatures and strong gradients almost closed magnetic field structures can hinder the TC. So, to describe SADs embedded in hot fans we propose the specific magnetic field configuration must have a fundamental role as a thermal insulator.

In this work, the scenario proposed in Paper I is revised, considering anisotropic TC in a turbulent magnetized fan. To summarize, the aim of this study is to address the following questions: how is it possible that SADs resist the thermal diffusion and are observed in hot fan regions? In fact, perturbations associated with typical SADs, -of sizes of various $\mathrm{Mm}$, typical coronal ion number densities of $\sim 10^{9} \mathrm{~cm}^{-3}$ and temperatures as high as $\sim 10$ MK- will fade away in times (of a few seconds) that are at least two orders of magnitude smaller than a typical SAD lifetime. Could a suitable magnetic field configuration avoid the thermal diffusion acting on the SADs' structures?

\section{The Model}

We integrate the ideal MHD equations considering the anisotropic heat flux and a solenoidal driving force in order to lead the plasma to a turbulent state. As here we would like to study how SADs are affected by TC in the framework of our SAD model (Costa et al. 2009; Cécere et al. 2012), now we choose (withing the ranges of observational data) a set of plasma parameters where the TC is more efficient than in Paper I. We use the set of values given in Table 1.

To numerically reproduced a detectable SAD, we assume that the following requirements have to be satisfied: 1) the EM contrast between the subdense cavity and its background has to be a factor $\gtrsim 4$ (Savage et al. 2012); and 2) the subdense cavity obtained must last at least one minute in the turbulent hot background. We consider valid the approximation of optically thin plasmas.

In order to estimate the importance of some physical processes we consider the actual set of plasma parameters, therefore the conduction, radiative and magnetic reconnection timescales (Paper I) give $t_{\text {cond }} \sim 0.5 \mathrm{~s}, t_{\text {rad }} \sim 10^{3} \mathrm{~s}$ and $t_{\text {rec }} \sim 10^{3} \mathrm{~s}$, respectively. Note that only the TC is able to modify SADs observability in times comparable with their lifetimes. On the other hand, we showed in Paper I that for relatively dense fans of $n_{i}=2 \times 10^{10}$ $\mathrm{cm}^{-3}$, and large SAD characteristic longitudes of $L=12 \mathrm{Mm}$, and ideal description of the problem could be possible because TC, radiative losses and reconnection releases of heat can compensate each other. However, SAD observations also report sizes of $\sim 1 \mathrm{Mm}$ remaining visible for several minutes, which cannot be reproduced by our model including TC, because their $t_{\text {cond }}$ values are even shorter. Thus, unless a mechanism acts to inhibit 
the strong TC diffusion, the subdense cavities will not last for times comparable with SAD observations.

We simulate the triggering of bursty reconnections that lead to the generation of SADs by applying instantaneous thermal pressure pulses, not modifying the density at the pulse region. Consequently the SAD temperature is larger than its neighborhood values, resulting in an almost total pressure equilibrium between the SAD structure and its vicinity. Our two-dimensional results seem to disagree with McKenzie \& Savage (2009), who suggested that the SAD collapse is avoided due to higher internal values of the magnetic pressure. Three-dimensional simulations are required to confirm this conclusion. Due to the simplified SAD setup proposed here (where the coronal medium standing above the fan is not modeled) we only consider the situation where SADs are triggered already inside the fan. Hanneman \& Reeves (2014) pointed out that SADs are colder than the fan but hotter than the coronal external medium. In the framework of our model, we believe that setting up a more realistic magnetic field configuration, with SADs triggered in the upper medium and then descending into the fan, might result in SADs with internal temperatures higher than the external medium but colder than the fan.

The purpose of the present work is to investigate the effectiveness of magnetic field lines in enveloping a $\mathrm{SAD}$, providing to it thermal insulation from its surroundings and allowing an explanation of the observed SAD characteristics.

\section{Numerical code and initial conditions}

The numerical simulations are performed with the FLASH code (Fryxell et al. 2000, release 4.2.2) solving the compressible MHD equations with the unsplit staggered mesh algorithm (Lee et al. 2009). We use a uniform 2D Cartesian grid with $300^{2}$ cells. The fan physical domain is set up to $(15,15) \mathrm{Mm}$, with the $y$-coordinate pointing radially from the Sun center (neglecting the curvature), and the $x$-coordinate parallel to the surface of the Sun. Periodic conditions are considered at all boundaries.

The simulation is carried on in two stages. As turbulent fans seem to be the natural site for the development of SADs (McKenzie 2013; Scott et al. 2016), the first is the turbulent stage where a turbulent state is obtained from initially homogeneous conditions (second row of Table 1) through the action of a solenoidal driving force. In order to do this we use the method of Federrath et al. (2010) (FromFile implementation of the Stir unit in the FLASH code). The first stage ends when the averaged quantities become stable, meaning that the system has reached a stationary state and has a 2D Kolmogorov-like spectrum. The plasma parameters showed in the third row of Table 1 correspond with the end of this stage. This turbulent state is the initial condition of the fan (second) stage. Here we turn off the stirring force, leaving the turbulence to gradually decay, and turn on the anisotropic TC. We also add a shear resembling the action of outflows coming from above the fan. At an early time of this stage we impose four bursty reconnection pulses. The turbulent stage occupies the time interval $t_{\text {turb }}=0-300 \mathrm{~s}$ while the fan stage takes 100 additional seconds $\left(t_{f a n}=300-400 \mathrm{~s}\right)$. The shear perturbation is instantaneously applied in the $y$-component of the velocity in the form

$$
v_{y}(t, x, y)= \begin{cases}v_{y}(t, x, y)-v_{s h} & \text { if } x<0, \\ v_{y}(t, x, y) & \text { if } x \geqslant 0\end{cases}
$$

at time $t=303.8 \mathrm{~s}$ and with $v_{s h}=1.5 \times 10^{7} \mathrm{~cm} \mathrm{~s}^{-1}$. Finally the four bursty reconnection pulses are instantaneously applied at time $t=305.9 \mathrm{~s}$, with diameters $L=1.2 \mathrm{Mm}$, labeled as $(A), \quad(B), \quad(C), \quad(D)$, centered at $\left(-3.0 \times 10^{8},-4.9 \times 10^{8}\right) \mathrm{cm}$, 

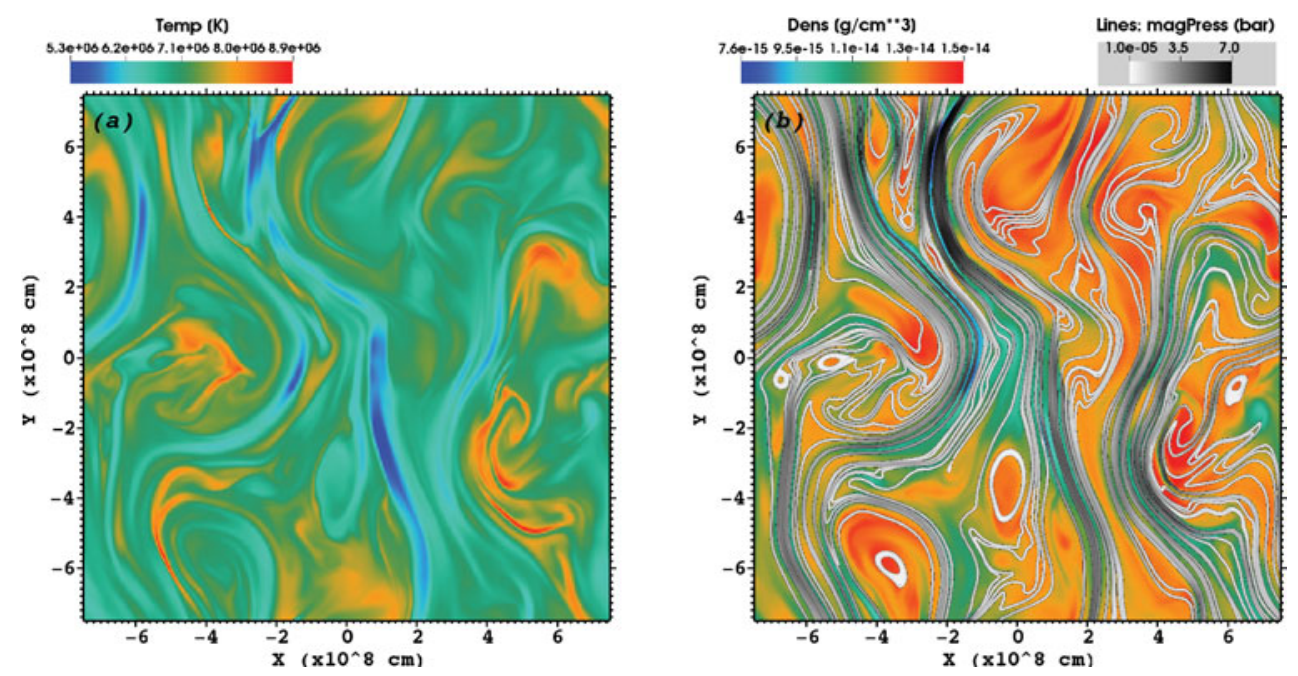

Figure 1. (a) The temperature pattern at an evolved time during the turbulent stage, at $t=290$ s. (b) Same as (a) but for the density superimposed with magnetic field lines. This is almost the initial condition for the fan stage. The color of the field lines indicate the magnetic pressure.

$\left(-9.6 \times 10^{6},-3.1 \times 10^{8}\right) \mathrm{cm},\left(5.3 \times 10^{7}, 5.5 \times 10^{8}\right) \mathrm{cm}$, and $\left(5.1 \times 10^{8}, 4.3 \times 10^{8}\right) \mathrm{cm}$, and with pressure contrasts of $\Delta P / P=(5,4,5,4)$, respectively.

In order to include the anisotropic TC a new module in the FLASH code was developed.

\section{Results and Discussion}

The driving force is turned off when the stationary state is reached and the final temperature is $\sim 7 \mathrm{MK}$, the stipulated background value. This state is considered the physical fan initial condition. While turbulence is included because it seems observationally established for fan descriptions, the consideration of downward velocity shears, that give account of typical SAD velocities, seem necessary due to the action of jets and outflows coming from above the CS. In addition, shears usually lead to Kelvin-Helmholtz instabilities favoring the reconnection and the production of isolated magnetic islands within the fan (Nakamura et al. 2008).

Figure 1(a)-(b) shows the temperature and density patterns almost at the end of the turbulent stage. Note that, the resulting magnetic topology organizes the plasma in vortical regions separated by strands formed by accumulations of magnetic field lines. These strand regions correspond to low internal values of temperature and density where the low gas pressure is compensated by large magnetic pressure values (McKenzie 2013). Also, vortices of approximately closed magnetic topologies have almost homogeneous internal density and temperature distributions. We call islands to vortices totally enclosed by magnetic field lines. As in McKenzie (2013) and in Scott et al. (2016), initially the fan has an average value of $\beta \gtrsim 1 \sim 28$. With this value of $\beta$ the magnetic forces do not play a dominant role in the turbulent dynamics. Thus, the observation of turbulent features seems a sign of relatively large values of $\beta$.

Finally, considering anisotropic TC, we apply pressure pulses near the vortices, the candidates to thermally isolate SADs. For simplicity we are not simulating the entrance of SADs to the fan. SADs are supposed to propagate into the fan with motions that become wrapped up by the magnetic field lines or that, eventually, they originate due to local reconnections inside the fan when field lines of different polarities approach enough. 

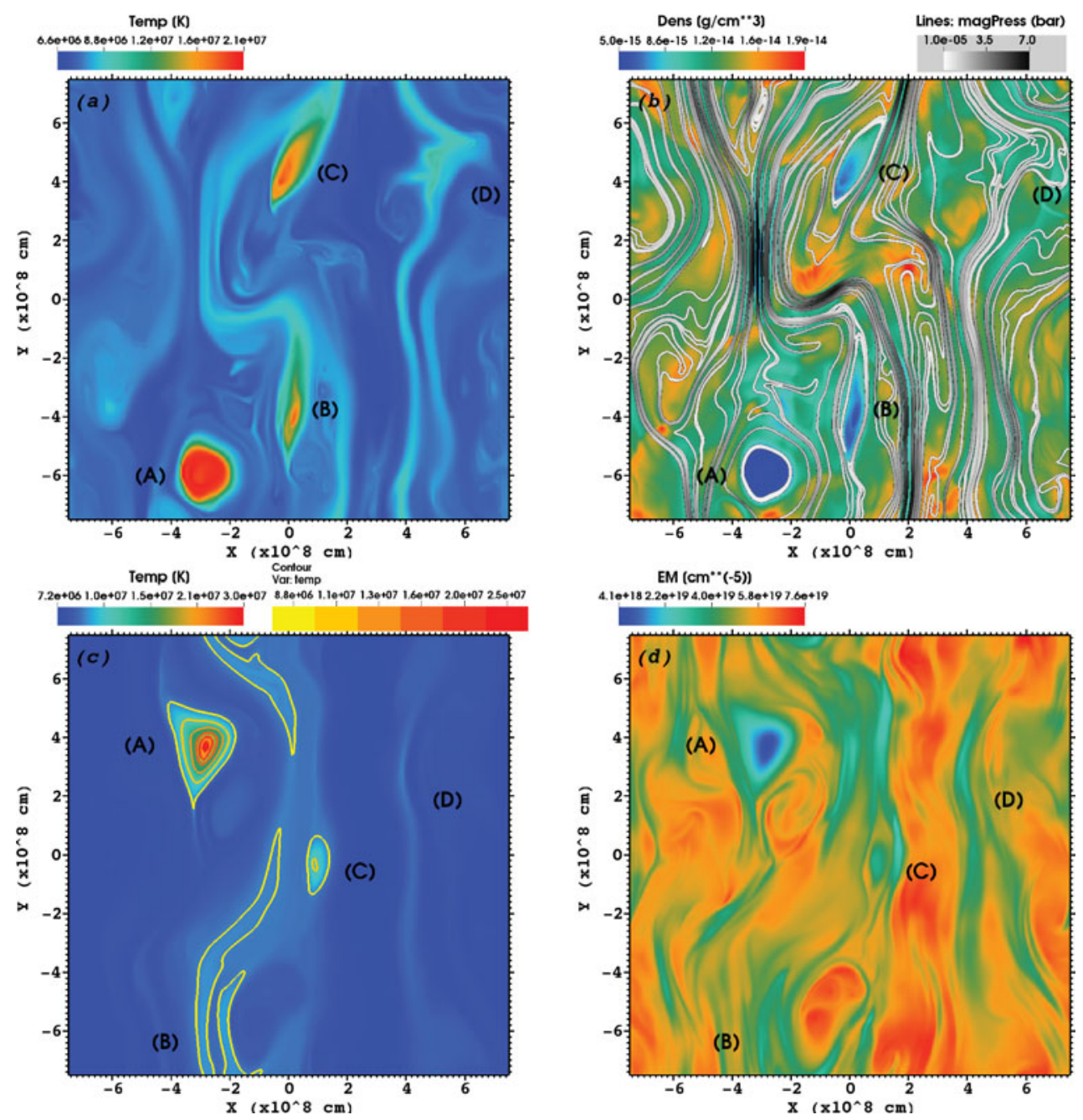

Figure 2. (a) The temperature pattern at $t=315 \mathrm{~s},(A),(B),(C)$ and $(D)$ are cavities triggered at $t=305.9$ s. (b) Same as (a) but for the density with magnetic field lines superimposed. (c) Same as (a) but one minute later, at $t=375 \mathrm{~s}$. (d) EM, also at $t=375 \mathrm{~s}$. Only cavity $(A)$ has an enough EM contrast of $\gtrsim 4$, while $(B)$ was scribbled, $(C)$ smoothed out and $(D)$ faded away.

The temperature and density patterns at time $t=315 \mathrm{~s}$ are shown in Fig. 2(a-b). The resulting pulses are labeled by $(A),(B),(C)$ and $(D)$. The same evolved patterns but a minute later, at $t=375 \mathrm{~s}$, are shown in Fig. $2(\mathrm{c}-\mathrm{d})$ for the temperature, with superimposed temperature contours, and for the emission measure (EM). If during their evolution the SAD candidates are not sufficiently enveloped by the magnetic field the EM contrast rapidly diminishes before reasonable observational times. Thus, features $(B),(C)$ and $(D)$ can not be identified as SADs, in accordance with our stipulations. At this moment only a slight hot subdense lane of $(D)\left(x \approx 4 \times 10^{8} \mathrm{~cm}\right)$ and a smooth trace of $(B)$ remain. Note that only $(A)$ is included inside an island where the temperature gradient is perpendicular to the magnetic field lines. The differences between the dynamical evolutions of features $(A)-(D)$, mainly resides in the capability of the magnetic field lines to thermally isolate them during their time evolution. Only cavity $(A)$ maintains an adequate EM contrast for times comparable with SAD observations. 


\section{Conclusions}

Several scenarios were proposed to give account of the origin and prevailing dynamics of subdense dark trails coming from the upper and cooler background corona into fan regions above flaring arcades. In previous works we proposed that these supra-arcade downflows (SADs) are subdense cavities created by nonlinear waves, lasting for times comparable with observations and triggered by bursty localized reconnections. Small SADs moving in hot fans $(T \gtrsim 7 \mathrm{MK})$ with large temperature gradients should be diffused on timescales shorter than their observed lifetimes. Thus, thermal conduction effects must be considered. We implemented the anisotropic thermal conduction to analyze different plasma conditions that make possible the survival of SADs avoiding the strong thermal damping. Here we considered SADs as voided cavities with diameters of $\sim 1 \mathrm{Mm}$ (almost the smallest values observed), that can maintain an emission measure contrast $\gtrsim 4$ and that prevail for at least one minute in a hot $2 \mathrm{D}$ fan. We found that triggered subdense cavities moving in a turbulent vortical magnetized fan can be identified as SAD features if in their downward motion they become enveloped by the magnetic field in such a way that heat conduction is inhibited. We also found that an increase of magnetic island production, where SADs are completely thermally isolated, reinforces the lifetime duration of SADs. Islands are expected due to shears produced by upper current sheet outflows and jets that lead to Kelvin-Helmoholtz instabilities allowing the reconnection of close enough magnetic field lines. The large fan values of $\beta$ found reinforces the turbulence development, i.e., the larger the $\beta$ values, the gas pressure more efficiently will bend the magnetic field lines. This allows the plasma evolves into highly rolled-up vortices and totally isolated islands. We conjecture that the conjunction of turbulent motions and large values of $\beta$ are a necessary requirement for the observation of small SADs in hot fans.

The software used in this work was in part developed by the ASC/Alliance Center for Astrophysical Thermonuclear Flashes at the University of Chicago.

\section{References}

Asai, A., Yokoyama, T., Shimojo, M., \& Shibata, K. 2004, ApJL, 605, L77

Aschwanden, M. J. 2005, "Physics of the Solar Corona. An Introduction with Problems and Solutions" (Praxis Publishing Ltd., 2nd ed.-2005)

Cécere, M., Schneiter, M., Costa, A., Elaskar, S., \& Maglione, S. 2012, ApJ, 759, 79

Cécere, M., Zurbriggen, E., Costa, A., \& Schneiter, M. 2015, ApJ, 807, 6

Chen, C. J. \& Lykoudis, P. S. 1972, SoPh, 25, 380

Costa, A., Elaskar, S., Fernández, C. A., \& Martínez, G. 2009, MNRAS, 400, L85

Federrath, C., Roman-Duval, J., Klessen, R., \& Schmidt, W., Mac Low 2010, A\&A, 512, A81

Fryxell, B., Olson, K., Ricker, P., et al. 2000, ApJS, 131, 273

Hanneman, W. J. \& Reeves, K. K. 2014, ApJ, 786, 95

Innes, D. E., McKenzie, D. E., \& Wang, T. 2003, SoPh, 217:247-265

Innes, D. E., Guo, L.-J., Bhattacharjee, A., Huang, Y.-M., \& Schmit, D. 2014, ApJ, 796, 27

Lazarian, A. \& Vishniac, E. T. 1999, ApJ, 517, 700

Lee, D., Deane, A. E., \& Federrath, C. 2009, AstroSocPacific Conference Series, Vol. 406, 243

McKenzie, D. E. \& Savage, S. L. 2009, ApJ, 697, 1569

McKenzie, D. E. 2013, ApJ, 766, 39

Nakamura, T. K. M., Fujimoto, M., \& Otto, A. 2008, JGRA, 113, A09204

Savage, S. L. \& McKenzie, D. E. 2011, ApJ, 730, 98

Savage, S. L., McKenzie, D. E., \& Reeves, K. K. 2012, ApJL, 747, L40

Scott, R. B., McKenzie, D. E., \& Longcope, D. W. 2016, ApJ, 819, 56

Spitzer, L. 1962, "Physics of Fully Ionized Gases" (New York: Interscience, 2nd ed.-1962) 